NBER WORKING PAPER SERIES

\title{
GENERALIZED DISAPPOINTMENT AVERSION AND ASSET PRICES
}

\author{
Bryan R. Routledge \\ Stanley E. Zin \\ Working Paper 10107 \\ http://www.nber.org/papers/w10107 \\ NATIONAL BUREAU OF ECONOMIC RESEARCH \\ 1050 Massachusetts Avenue \\ Cambridge, MA 02138 \\ November 2003
}

Thanks to David Backus, Chew Soo Hong, Larry Epstein, Rick Green, Bart Lipman, Uday Rajan, Jacob Sagi, and seminar participants at Carnegie Mellon University, Simon Fraser University, University of British Columbia, University of Washington, and SED 2003 for helpful comments. The views expressed herein are those of the authors and not necessarily those of the National Bureau of Economic Research.

(C)2003 by Bryan R. Routledge and Stanley E. Zin. All rights reserved. Short sections of text, not to exceed two paragraphs, may be quoted without explicit permission provided that full credit, including (C) notice, is given to the source. 
Generalized Disappointment Aversion and Asset Prices

Bryan R. Routledge and Stanley E. Zin

NBER Working Paper No. 10107

November 2003

JEL No. G0, G1, D8

\begin{abstract}
We provide an axiomatic model of preferences over atemporal risks that generalizes Gul's disappointment aversion model by allowing risk aversion to be "first order" at locations in the state space that do not correspond to certainty. Since the lotteries being valued by an agent in an assetpricing context are not typically local to certainty, our generalization, when embedded in a dynamic recursive utility model, has important quantitative implications for financial markets. We show that the state-price process, or asset-pricing kernel, in a Lucas-tree economy in which the representative agent has generalized disappointment aversion preferences is consistent with the pricing kernel that resolves the equity-premium puzzle. We also demonstrate that a small amount of conditional heteroskedasticity in the endowment-growth process is necessary to generate these favorable results. In addition, we show that risk aversion in our model can be both state-dependent and countercyclical, which empirical research has demonstrated is necessary for explaining observed assetpricing behavior.
\end{abstract}

Bryan R. Routledge

Graduate School of Industrial Administration

Carnegie Mellon University

Pittsburgh, PA 15213-3890

rout@andrew.cmu.edu

Stanley E. Zin

Graduate School of Industrial Administration

Carnegie Mellon University

Pittsburgh, PA 15213-3890

and NBER

zin@cmu.edu 


\section{Introduction}

The observed facts of aggregate risks and asset prices in the post-war US economy have led researchers to explore models of intertemporal preferences that generalize the well-developed time-additive-expected-utility specification used in the asset-pricing economy of Lucas (1978). In particular, models that allow for counter-cyclical risk aversion have been shown to perform much better than the standard model (see, for example, Campbell and Cochrane (1999), Gordon and St-Amour (2000), and Barberis, Huang, and Santos (2001)). ${ }^{1}$ While these models all seem reasonable, the lack of an axiomatic foundation makes it difficult to assess their structural integrity and to impose a homothetic structure on preferences. ${ }^{2}$ In this paper, we adopt an axiomatic approach to the specification of risk preferences. Our axioms result in a one-parameter extension of the Gul (1991) disappointment aversion utility function. Our extension to Gul results in preferences that have the desirable property that, when embedded in a dynamic asset-pricing economy, effective risk aversion can counter-cyclical. Moreover, the state-prices that our risk-preference assumptions generate are precisely those that rationalize the equity-premium puzzle of Mehra and Prescott (1985).

Melino and Yang (2002) provide a useful characterization of the need for counter-cyclical risk aversion by directly calculating the pricing kernel that resolves the equity-premium puzzle. Setting the first two moments of consumption growth equal to their historical estimates in the U.S., $E[x]=1.018$, $V[x]=0.036^{2}$, and Corr $\left[x_{t}, x_{t-1}\right]=-0.14$, consumption growth is calibrated as a two-state Markov process with the state space for growth rates given by

\footnotetext{
${ }^{1}$ Campbell and Cochrane (1999) capture counter-cyclical risk aversion through an external habit with a time-varying "habit sensitivity." Barberis, Huang, and Santos (2001) and Barberis and Huang (2001) create this with an ad hoc utility function that features a timevarying loss aversion (a direct disutility from negative stock market returns). Gordon and St-Amour (2000) take a more direct approach and calibrate a time-varying risk aversion.

${ }^{2}$ To compensate for the lack of homotheticity, Barberis, Huang, and Santos (2001), for example, introduce a time-varying scaling to the loss aversion to force stationarity. Similarly, the non-stationarity of Gordon and St-Amour (2000) preferences restricts their application to a finite-horizon economy.
} 
$x_{L}=1.018-0.036$ and $x_{H}=1.018+0.036$, and transition probabilities

$$
\Pi=\left[\begin{array}{ll}
0.43 & 0.57 \\
0.57 & 0.43
\end{array}\right]
$$

Melino and Yang then choose an asset-pricing kernel represented by a stochastic process $M_{t}$, that exactly matches the historical means and variances of equity and bond returns of $E[R]=1.07, V[R]=0.165^{2}, E[r]=1.008$, and $V[r]=0.056^{2}$, and satisfies the usual arbitrage-free pricing condition, $E_{t}\left[M_{t+1} R_{t+1}^{i}\right]=1$. The resulting just-identified pricing kernel is given by the 2-by-2 matrix:

$$
\left[\begin{array}{ll}
M_{L L} & M_{L H} \\
M_{H L} & M_{H H}
\end{array}\right]=\left[\begin{array}{ll}
1.862 & 0.244 \\
1.127 & 0.949
\end{array}\right] .
$$

The first row of equation (2) is the kernel when the current state is a recession denoted as $L$ (i.e., low consumption growth), the second row is the kernel conditional on being in an expansion denoted as $H$ (i.e., high consumption growth). It is immediately apparent why a traditional time-additive expected utility function will have difficulty matching historical asset-return behavior. Note that the transition probabilities are not dramatically different in the recession and expansion state $\left(\pi_{L L} / \pi_{L H}=0.75\right.$ and $\left.\pi_{L L} / \pi_{L H}=1.33\right)$. However, the pricing kernel is dramatically different across the two states. In the $x_{t}=L$ state, there is a large difference in the price of $\$ 1$ contingent on $x_{t+1}=L$ versus $x_{t+1}=H$, suggesting a high degree of risk aversion in the standard model $\left(M_{L L} / M_{L H}=7.63\right)$. However, in the $x_{t}=H$ state the price of $\$ 1$ in $x_{t+1}=L$ and $x_{t+1}=H$ are very similar, suggesting a very low degree of risk aversion in the standard model $\left(M_{L L} / M_{L H}=1.18\right)$. To capture the dynamics of asset returns requires an effective risk-aversion that is both state dependent and counter-cyclical (i.e., higher risk aversion in the low-growth state).

Thus far, axiomatic preferences that can accommodate experimental results have had little success in capturing the dynamic pattern of asset prices displayed in equation (2). For example, Gul (1991) is an axiomatic characterization of preferences that allows for an asymmetric treatment of the outcomes 
of a lottery based on where the outcomes lie relative to an implicit certainty equivalent. In essence, outcomes below the certainty equivalent are disappointing and, hence, receive relatively more weight in the utility calculation of a disappointment-averse agent. Gul's relaxation of the von Neumann and Morgenstern (1947) independence axiom yields preferences that are consistent with the Allais Paradox. For portfolio choice and asset pricing, the important manifestation of these preferences is "first-order" risk-aversion (Epstein and Zin (1990), Ang, Bekaert, and Liu (2002)). That is, for gambles local to certainty, risk aversion is first-order and indifference curves in state-space have a kink at certainty. Unfortunately, Gul preferences have little success in explaining asset returns. In a Lucas-tree endowment economy in which the representative agent has disappointment aversion the first-order risk aversion is not of first-order importance since the endowment, and hence the equilibrium optimal consumption plan, is not local to certainty. Analogous to the conclusion of Mehra and Prescott (1985), the calibrated disappointment aversion needed to generate a large equity premium seems unreasonably high and the model is still unable to capture many of the important patterns in the historical asset returns (see Epstein and Zin (2001)).

In this paper, we provide an axiomatic model of preferences over atemporal risks that generalizes the disappointment aversion model. We extend the Gul (1991) model by modifying the definition of a disappointing outcome to allow for a focus on more extreme outcomes (i.e., generalized disappointment aversion). That is, an outcome is disappointing only when it is sufficiently far from the implicit certainty equivalent. ${ }^{3}$ This moves the locus of first-order risk aversion away from certainty. The advantage of these preferences is that we can disentangle the slope of preferences at the endowment from the level of the certainty equivalent. The slope of the indifference curve determines the price of risk while the level of the certainty equivalent drives the risk-free interest rate. Because our approach is axiomatic, we can maintain the analytic

\footnotetext{
${ }^{3}$ In Gul (1991) and our extension, the reference point that defines disappointment is internal to the gamble being considered. In contrast, Sagi (2003) considers axioms that allow an external reference point as in models of loss aversion.
} 
appeal of properties like homotheticity. However, the structure placed on preferences by other axioms (e.g., continuity and monotonicity) restrict the degree of first-order risk aversion. In state-space, a kink away from certainty must be less severe. To investigate the quantitative implications of these preferences, we embed our model of atemporal preferences in the recursive utility model of Epstein and Zin (1989). In this setting, our preference specification yields tractable Euler equations which facilitate calibration and empirical analysis.

Our calibration results show that the equilibrium asset returns in the Mehra and Prescott (1985) version of a Lucas-tree economy in which the representative agent has generalized disappointment aversion, is not much of an improvement over Gul's disappointment aversion, which is not much of an improvement over standard expected utility. That is, none of these models can produce the Melino and Yang kernel of equation (2). On the other hand, a small departure from the Mehra-Prescott calibration that introduces a small amount of conditional heteroskedasticity, while maintaining all other features of their calibration, creates an environment in which generalized disappointment aversion preferences are able to capture the state-dependent risk aversion described above, and exactly match the pricing-kernel that resolves the equitypremium puzzle.

The paper is organized by starting with the axiomatic foundations of generalized disappointment aversion (GDA) in Section 2. Along with the axioms, several of the properties of the resulting GDA utility function are presented. Section 3 presents the infinite horizon, Lucas-tree economy using the GDA preferences along with a calibration to the Melino and Yang kernel of equation (2). Section 3.2 explains why GDA preferences are not able to improve on existing models in replicating the observed data. We then propose a small change in the calibration to introduce conditional heteroskedasticity and demonstrate that GDA preferences can dramatically improve the performance of the assetpricing model. Section 4 points to directions for future research and concludes. 


\section{Generalized Disappointment Aversion}

In this section, we present the axioms which are necessary and sufficient for a generalization of Gul (1991) disappointment aversion. Some readers may prefer to move directly to the asset pricing model of Section 3. In that section, we use a one-parameter extension of Gul disappointment aversion where the functional form for risk preferences is a linearly homogeneous certainty equivalent $\mu(p)$ for lottery $p$ that solves

$$
u(\mu(p))=\sum_{x_{i} \in X} p\left(x_{i}\right) u\left(x_{i}\right)-\beta \sum_{x_{i} \leq \delta \mu(p)} p\left(x_{i}\right)\left(u(\delta \mu(p))-u\left(x_{i}\right)\right)
$$

where

$$
u(x)= \begin{cases}\frac{x^{\alpha}-1}{\alpha} & \alpha \leq 1, \alpha \neq 0 \\ \log (x) & \alpha=0\end{cases}
$$

$\alpha$ is a risk aversion parameter and $\beta \geq 0$, and $\delta \leq 1$ capture disappointment aversion. If $\beta=0$, the preferences are equivalent to expected utility with constant relative risk aversion parameter $\alpha$. If $\delta=1$, the preferences are equivalent to Gul disappointment aversion. Disappointment averse preferences imply a penalty for outcomes below the certainty equivalence when $\beta>0$. We generalize Gul preferences by modifying the definition of disappointment. Preferences with $\delta<1$ capture non-central disappointment aversion by moving the disappointment cut-off. Outcomes are disappointing only if they lie sufficiently far below the certainty equivalent. As mentioned previously, this allows for first-order-risk-aversion effects away from certainty, and is the key feature of our calibrated asset-pricing economy. The axiomatic derivation of this utility representation, its properties, and some extensions are developed in the remainder of this section. 


\subsection{Maintained Axioms}

Let $X=\left[x_{0}, x^{0}\right]$ be the set of monetary outcomes and $\mathcal{L}$ be the set of finitesupport lotteries on $X$. Lotteries that assign probability one to a single $x \in X$ are denoted simply as $x$. Let $\preceq$ be a binary relation on $\mathcal{L}$ using the standard notation of $p \succ q$ means $p$ is strictly preferred to $q, p \succeq q$ denotes weakly preferred, and $p \sim q$ denotes indifference.

Axiom 0 - Monotonicity: For $x, y \in X, x \succ y$ if and only if $x>y$

Axiom 1 - Preference Relation: $\preceq$ is complete and transitive.

AxIOM 2 - Continuity: For all $p \in \mathcal{L}$, the sets $\{q \mid q \succeq p\}$ and $\{q \mid p \succeq q\}$ are closed. $^{4}$

These axioms imply that the certainty equivalent $\mu: \mathcal{L} \rightarrow X$ (i.e, $\mu(p) \sim p$ ) is a well defined function.

\subsection{Disappointment Aversion}

The two central axioms in Gul are Weak Independence and Symmetry. Both axioms rely on the definition of an elation/disappointment decomposition. We modify the decompositions used in Gul in a straightforward fashion and define sets $B_{\delta}$ and $W_{\delta}$ as follows.

$$
\begin{aligned}
& B_{\delta}(p)=\{q \mid x \in \operatorname{supp}(q) \rightarrow x \geq \delta \mu(p)\} \\
& W_{\delta}(p)=\{q \mid x \in \operatorname{supp}(q) \rightarrow x \leq \delta \mu(p)\}
\end{aligned}
$$

where $\delta \leq 1$ is a parameter. Next, define decompositions of a lottery as $\alpha \in[0,1]$ and $q, r \in \mathcal{L}$ such that $p=\alpha q+(1-\alpha) r$ with $q \in B_{\delta}(p)$ and $r \in W_{\delta}(p)$. The lottery $p$ is decomposed into two lotteries. Lottery $q$ has zero probability on all elements below the threshold $\delta \mu(p)$ while lottery $r$ has zero

\footnotetext{
${ }^{4}$ Using the topology generated by the $L^{1}$ metric. See Gul (1991).
} 
probability for outcomes above the threshold. The parameter $\delta$ determines the location of the disappointment threshold. By focusing on the certainty equivalent, Gul's disappointment averse preferences have a central measure of disappointment. This is captured here with $\delta=1$. Our generalization allows for a non-central, $\delta<1$, characterization of disappointment.

The expected utility axiom of independence states that $p_{1} \succ p_{2}$ implies $\lambda p_{1}+(1-\lambda) x \succ \lambda p_{2}+(1-\lambda) x$. This axiom is frequently violated in experiments. The Allais Paradox, for example, is perhaps the most familiar violation (see, Allais (1979) Conlisk (1989) Machina (1987)). Following Gul, the decomposition facilitates characterizing preferences that are averse to disappointment by overweighting (relative to their probabilities) outcomes below the disappointment threshold. This asymmetric weighting is a weakening of the independence axiom. Specifically, the independence axiom need not hold if $p_{1}$ and $p_{2}$ assign different probability weight to disappointing outcomes. This is stated in axiom 3 .

AxIom $3-\delta$-Weak Independence: $\quad$ For all $p \in \mathcal{L}$ and $\lambda \in(0,1) \mu(p) \sim$ $\lambda p+(1-\lambda) \mu(p)$. There exists a $\delta \leq 1$ such that for all $p_{1}, p_{2} \in \mathcal{L}$ with $p_{1} \succ p_{2}, \lambda \in(0,1)$, and $x \in X, \lambda p_{1}+(1-\lambda) x \succ \lambda p_{2}+(1-\lambda) x$ if (1) $W_{\delta}\left(p_{j}\right) \cap W_{\delta}\left(\lambda p_{j}+(1-\lambda) x\right)=\varnothing$ for $j=1,2$, or (2) there exits an $\alpha \in[0,1]$ and $q_{j}, r_{j} \in \mathcal{L}$ such that for $j=1,2$

$$
\begin{aligned}
& p_{j}=\alpha q_{j}+(1-\alpha) r_{j} \\
& q_{j} \in B_{\delta}\left(p_{j}\right) \cap B_{\delta}\left(\lambda p_{j}+(1-\lambda) x\right) \\
& r_{j} \in W_{\delta}\left(p_{j}\right) \cap W_{\delta}\left(\lambda p_{j}+(1-\lambda) x\right) .
\end{aligned}
$$

The axiom is the direct analog to Weak Independence in Gul. The axiom allows for preferences to not satisfy the independence axiom when disappointment is a concern. As with Gul, the decompositions of $p_{j}$ in equation (5) place the same probability, $1-\alpha$, on the disappointing outcomes. The restriction that $q_{j} \in B_{\delta}\left(p_{j}\right)$ and $q_{j} \in B_{\delta}\left(\lambda p_{j}+(1-\lambda) x\right)$ and, similarly $r_{j} \in W_{\delta}\left(p_{j}\right)$ and $r_{j} \in W_{\delta}\left(\lambda p_{j}+(1-\lambda) x\right)$ implies that mixing in the payoff, $x$, has not changed 
the nature of disappointment. We will refer to the Gul case of $\delta=1$ as Central Weak Independence and $\delta<1$ as Non-Central Weak Independence. With $\delta<1$, it is possible that no outcomes are disappointing, $W_{\delta}\left(p_{j}\right)=$ $W_{\delta}\left(\lambda p_{j}+(1-\lambda) x\right)=\varnothing$. In this case, the usual independence axiom holds. Lastly, the axiom requires preferences to satisfy the betweenness property $\mu(p) \sim \lambda p+(1-\lambda) \mu(p)$. This requirement is redundant in the case of $\delta=1$ (see Gul (1991)) and $\delta<\frac{x_{0}}{x^{0}}$ (see Property 5 below). However, it is required for intermediate values of $\delta$ to ensure preferences are linear in probabilities (i.e., linear indifference curves on a probability simplex).

The final axiom used by Gul is Symmetry. This axiom requires that outcomes above and below the disappointment cut-off be evaluated using the same outcome valuation function. The axiom is stated considering lotteries $p_{j}$ that have the property that all outcomes in the support of $p_{j}$ are disappointing in the lottery $\alpha x^{0}+(1-\alpha) p_{j}$ (recall $x^{0}$ is the largest possible lottery payoff) but not-disappointing in the lottery $\alpha p_{j}+(1-\alpha) x_{0}\left(x_{0}\right.$ is the worst possible payoff).

Axiom 4 - $\delta$-Symmetry: Given a $\delta$ from Axiom 3, for all $p_{1}, p_{2} \in \mathcal{L}$ and $\alpha \in[0,1]$ such that for $j=1,2, p_{j} \in B_{\delta}\left(\alpha p_{j}+(1-\alpha) x_{0}\right)$ and $p_{j} \in$ $W_{\delta}\left(\alpha x^{0}+(1-\alpha) p_{j}\right)$

$$
\begin{aligned}
& \alpha p_{1}+(1-\alpha) x_{0} \succeq \alpha p_{2}+(1-\alpha) x_{0} \quad \text { iff } \\
& \alpha x^{0}+(1-\alpha) p_{1} \succeq \alpha x^{0}+(1-\alpha) p_{2}
\end{aligned}
$$

Note the same disappointment cut-off, $\delta \mu$, is used in Axiom 4 as in Axiom 3.

\subsection{Preference Representation}

To characterize disappointment aversion preferences, it is helpful to start with the general class of preferences that are linear in probabilities (i.e., satisfy the 
betweenness property) and then consider the restrictions imposed by $\delta$-Weak Independence and $\delta$-Symmetry. Dekel (1986) and Chew (1989) characterize preferences that satisfy betweenness as a certainty equivalent $\mu(p)$ that solves $\sum_{x_{i} \in X} p\left(x_{i}\right) U\left(x_{i}, \mu(p)\right)=0$. As shown in the lemma in the appendix, we can define functions $u, D_{k}$, and $L_{k}$ (whose properties are stated in the appendix) that re-state this characterization as the function $M: \mathcal{L} \times X \rightarrow \mathbb{R}$, such that

$$
M(p, m)=u^{-1}\left(\sum_{x_{i} \in X} p\left(x_{i}\right) u\left(x_{i}\right)-\sum_{k=1}^{K} \beta_{k} \sum_{x_{i} \leq \Delta_{k}(m)} p\left(x_{i}\right) L_{k}\left(x_{i}, \Delta_{k}(m)\right)\right)
$$

Equation (7) defines the certainty equivalence of lottery $p$ as $\mu(p)=M(p, \mu(p))$ and $p \succeq q$ if and only if $\mu(p) \geq \mu(q)$. The function, $M(p, m)$, calculates the expected utility, using $u(\cdot)$, of lottery $p$ minus penalties, denoted $L_{k}(x, m)$, for outcomes that lie below the cut-offs $\Delta_{k}(m)$. Note $\beta_{k}=0$ for all $k$ is equivalent to expected utility. Gul's disappointment aversion has just one cut-off $\left(\beta_{k}=0\right.$ for all $\left.k>1\right)$ at $\Delta_{1}(m)=m$, and a linear penalty function $L_{1}(x, m)=u(m)-u(x)$.

THEOREM: $\preceq$ satisfies Axioms 0 -3 if and only if they are represented by $\mu(p)=M(p, \mu(p))$ in equation (7) with: $K=1, \Delta_{1}(m)=\delta m, L_{1}(x, \delta m)=$ $\ell(\delta m)-\ell(x)$ with $\ell(\cdot)$ a continuous increasing function. $\preceq$ satisfies Axioms $0-4$ if and only if in addition, $\ell(\cdot)$ is an affine transformation of $u(\cdot)($ e.g., $\ell(x)=u(x))$.

Proof: The Lemma in the see appendix shows that the betweenness property implies the functional form of equation (7). In addition, $\Delta_{k}(m)$ and $L_{k}\left(x, \delta_{k}(m)\right)$ are increasing in $x$ and decreasing in $m$, ensuring $\mu(p)=M(p, \mu(p))$ exists and is unique. Finally, $L_{k}\left(x, \Delta_{k}(m)\right)=0$ if $x=\Delta_{k}(m)$. For expositional purposes, consider $u(x)=x$ and $X=[0,1]$, since extending to general $u(x)$ and $X$ is straightforward. 
The $\delta$-Weak Independence axiom can be stated as

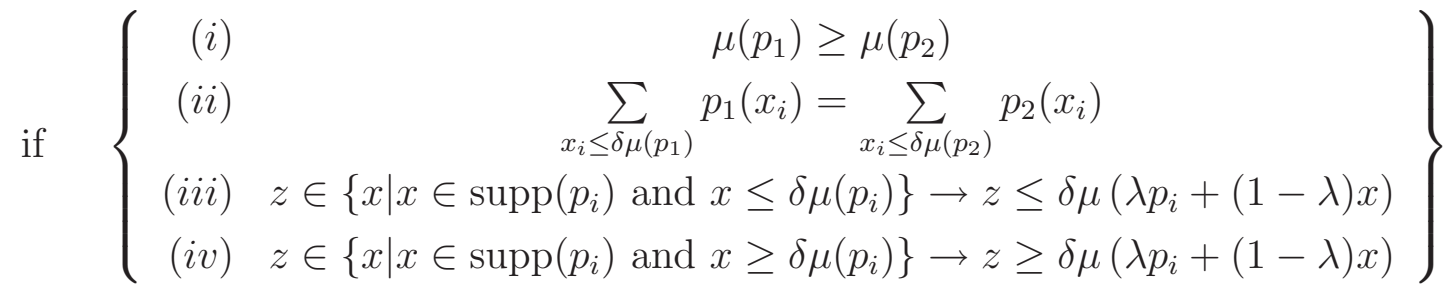

then $\quad \mu\left(\lambda p_{1}+(1-\lambda) x\right) \geq \mu\left(\lambda p_{2}+(1-\lambda) x\right)$.

Assume $p_{1}, p_{2}$, and $\lambda$ satisfy the necessary conditions of equation (8). For notation, let $\mu_{j}=\mu\left(p_{j}\right)$ and $\hat{\mu}_{j}=\mu\left(\lambda p_{j}+(1-\lambda) x\right)$. Consider equation (7) with one cut-off $(K=1)$.

$$
\begin{aligned}
\hat{\mu}_{j}= & \lambda \sum_{x_{i} \in X} p_{j}\left(x_{i}\right) x_{i}-\lambda \beta \sum_{x_{i} \leq \Delta\left(\hat{\mu}_{j}\right)} p_{j}\left(x_{i}\right) L\left(x_{i}, \Delta\left(\hat{\mu}_{i}\right)\right) \\
& +(1-\lambda) x-(1-\lambda) \beta L\left(x_{i}, \Delta\left(\hat{\mu}_{i}\right)\right) 1_{\left\{x \leq \Delta\left(\hat{\mu}_{j}\right)\right\}}
\end{aligned}
$$

where $1_{\{\cdot\}}$ is an indicator function. For $\beta>0$ and for arbitrary $\Delta(m)$ and $L(x, \Delta(m))$, it will not be the case that $\hat{\mu}_{1}>\hat{\mu}_{2}$. Satisfying Weak Independence requires that $\Delta(m)=\delta m$ (hence $K=1$ and there can be no other cut-offs). This implies

$$
\begin{aligned}
\hat{\mu}_{j}= & \lambda \mu_{j}+\lambda \beta\left(\sum_{x_{i} \leq \delta \mu_{i}} p_{j}\left(x_{i}\right) L\left(x_{i}, \delta \mu_{i}\right)-\sum_{x_{i} \leq \delta \hat{\mu}_{j}} p_{j}\left(x_{i}\right) L\left(x_{i}, \delta \hat{\mu}_{j}\right)\right) . \\
& +(1-\lambda)\left(x-\beta L\left(x, \delta \hat{\mu}_{j}\right) 1_{\left\{x \leq \delta \hat{\mu}_{j}\right\}}\right)
\end{aligned}
$$

By parts (iii) and (iv) of (8),

$$
\begin{aligned}
\hat{\mu}_{j}= & \lambda \mu_{j}+\lambda \beta\left(\sum_{x_{i} \leq \delta \mu_{j}} p_{j}\left(x_{i}\right)\left(L\left(x_{i}, \delta \mu_{j}\right)-L\left(x_{i}, \delta \hat{\mu}_{j}\right)\right)\right) \\
& +(1-\lambda)\left(x-\beta L\left(x, \delta \hat{\mu}_{j}\right) 1_{\left\{x \leq \delta \hat{\mu}_{j}\right\}}\right)
\end{aligned}
$$

Again, for arbitrary $L(x, \delta m)$ we can construct lotteries such that $\hat{\mu}_{2}>\hat{\mu}_{1}$ (violating weak independence). It must therefore be the case that the loss 
function is separable in $x$ and $\delta m$. The restriction that $L(x, \delta m)=0$ if $x=$ $\delta m$ means that an additively separable loss function must be of the form $L(m, x)=\ell(\delta m)-\ell(x)$, where $\ell(\cdot)$ is an increasing function. Inserting this into (9) and using, by part (ii) of (8) that $\sum_{x_{i} \leq \mu_{1}} p_{1}\left(x_{i}\right)=\sum_{x_{i} \leq \mu_{2}} p_{2}\left(x_{i}\right)=\alpha$ produces

$$
\hat{\mu}_{j}=\lambda \mu_{j}+\lambda \beta \alpha\left[\ell\left(\delta \mu_{j}\right)-\ell\left(\delta \hat{\mu}_{j}\right)\right]+(1-\lambda)\left(x-\beta\left[\ell\left(\delta \hat{\mu}_{j}\right)-\ell(x)\right] 1_{\left\{x \leq \hat{\mu}_{j}\right\}}\right)
$$

In this form, it is clear that if $\mu_{1}>\mu_{2}$ then $\hat{\mu}_{1}>\hat{\mu}_{2}$ satisfying weak independence.

Next consider the role of the symmetry axiom. For lotteries $p_{1}$ and $p_{2}$, let $\mu_{j b}=\mu\left(\alpha x^{0}+(1-\alpha) p_{j}\right)$ and $\mu_{j w}=\mu\left(\alpha p_{j}+(1-\alpha) x_{0}\right)$. We calculate these certainty equivalents next, using the fact that $p_{j} \in B\left(\alpha p_{j}+(1-\alpha) x_{0}\right)$ and $p_{j} \in W\left(\alpha x^{0}+(1-\alpha) p_{j}\right)$ imply that $\delta \mu_{j w}<z<\delta \mu_{j b}$ for all $z \in \operatorname{supp}\left(p_{j}\right)$.

$$
\begin{aligned}
\mu_{j w}=\alpha\left[\sum_{x_{i} \in X} p_{j}\left(x_{i}\right) x_{i}-\beta \sum_{x_{i} \leq \delta \mu_{j w}} p_{j}\left(x_{i}\right)\left(\ell\left(\delta \mu_{j w}\right)-\ell\left(x_{i}\right)\right)\right] \\
\quad+(1-\alpha)\left[x_{0}-\beta\left(\ell\left(\delta \mu_{j w}\right)-\ell\left(x_{0}\right)\right)\right] \\
=\alpha \sum_{x_{i} \in X} p_{j}\left(x_{i}\right) x_{i}+(1-\alpha)\left[w-\beta\left(\ell\left(\delta \mu_{j w}\right)-\ell\left(x_{0}\right)\right)\right]
\end{aligned}
$$

and

$$
\begin{aligned}
\mu_{j b} & =\alpha x^{0}+(1-\alpha)\left[\sum_{x_{i} \in X} p_{j}\left(x_{i}\right) x_{i}-\beta \sum_{x_{i} \leq \delta \mu_{j b}} p_{j}\left(x_{i}\right)\left(\ell\left(\delta \mu_{j b}\right)-\ell\left(x_{i}\right)\right)\right] . \\
& =\alpha x^{0}+(1-\alpha)\left[\sum_{x_{i} \in X} p_{j}\left(x_{i}\right) x_{i}+\beta \sum_{x_{i} \in X} p_{j}\left(\ell\left(x_{i}\right)\right)-\ell\left(\delta \mu_{j b}\right)\right]
\end{aligned}
$$

$\mu_{j w}$ depends on $p_{j}$ only through the first moment (the first term of equation $(10))$. However, $\mu_{j b}$ depends on the first moment of $p$ as well as moments generated by $\ell(\cdot)$ (note $\sum p_{j}\left(x_{i}\right) \ell\left(x_{i}\right)$ in equation (11)). Symmetry requires that $\mu_{1 b} \geq \mu_{2 b}$ if and only if $\mu_{1 w} \geq \mu_{2 w}$. The only way this condition can be satisfied for all lotteries is for the penalty function to be affine, e.g., $\ell(x)=x$ or more generally, $\ell(\cdot)=u(\cdot)$ 


\subsection{Properties of Generalized Disappointment Aversion}

The preference representation in the Theorem suggest two directions for extending Gul's disappointment aversion. We can consider central $(\delta=1)$ and non-central $(\delta<1)$ definitions of disappointment. In addition, we can relax the symmetry axiom by adopting a non-linear penalty function, $\ell(\cdot)$. Unfortunately, as we show below, symmetry is necessary for linear homogeneity of the certainty equivalent. To discuss the properties of generalized disappointment aversion, consider

$$
\mu(p)^{\alpha}=\sum_{x_{i} \in X} p\left(x_{i}\right) x_{i}^{\alpha}-\beta \sum_{x_{i} \leq \delta \mu(p)} p\left(x_{i}\right)\left((\delta \mu(p))^{\alpha \sigma}-x_{i}^{\alpha \sigma}\right)
$$

where $\sigma=1$ satisfies the Symmetry axiom.

PROPERTY 1: The certainty equivalent, $\mu(p)$, implicitly defined by equation (12) is unique and is a continuous function of $p$.

Although this fact is a direct implication of Theorem 1, it is helpful to note that finding the certainty equivalent is a simple fixed-point calculations. Consider the (not implicit) function (the CRRA analog to equation (7))

$$
M(p, m)^{\alpha}=\sum_{x_{i} \in X} p\left(x_{i}\right) x_{i}^{\alpha}-\beta \sum_{x_{i} \leq \delta m} p\left(x_{i}\right)\left((\delta m)^{\alpha \sigma}-x_{i}^{\alpha \sigma}\right) .
$$

$M$ is a continuous function that is (weakly) decreasing in $m$. For continuity, it is important to note that at the disappointment threshold of $x_{i}=\delta \hat{\mu}$, $(\delta m)^{\alpha \sigma}-\left(x_{i}\right)^{\alpha \sigma}=0$. Suppose $\delta M(p, m)<m$ for all $m$. In this case, $p$ has no disappointing outcomes and the certainty equivalent is identical to expected utility, $\mu(p)^{\alpha}=\sum_{x_{i} \in X} p\left(x_{i}\right) x_{i}^{\alpha}$. Alternatively, there exists a unique fixed point $\mu(p)=M(p, \mu(p))$ which is continuous by the implicit function theorem.

Property 2: $\mu(x)=x$.

Simply verify that $M(x, x)^{\alpha}=x^{\alpha}$. 
PROPERTY 3: An increase in $\beta$ or $\delta$ increases risk aversion in that the certainty equivalent falls; i.e., $\partial \mu(p) / \partial \beta \leq 0$ and $\partial \mu(p) / \partial \delta \leq 0$.

This follows from equation (13). Note that $\partial M(p, m) / \partial \beta \leq 0$ and $\partial M(p, m) / \partial \delta \leq$ 0 for all $m$.

Property 4: If $\sigma \geq 1$, an increase in $\sigma$ increases risk aversion in that the certainty equivalent declines; i.e., $\partial \mu(p) / \partial \sigma \leq 0$

Similarly, this follows from equation (13).

PROPERTY 5: The preferences characterized by equation (3) are equivalent to expected utility if $\beta=0, \delta \leq \frac{x_{0}}{x^{0}}$, or $\sigma=0$.

It is obvious from the specifications in equation (3) that $\beta=0$ produces expected utility. $\delta \leq \frac{x_{0}}{x^{0}}$ implies that for all lotteries, $\delta \mu(p) \leq x_{0}$ so there are no lotteries with disappointing outcomes. That is, for all $p \in \mathcal{L}, \sum_{x_{i} \leq \delta m} p\left(x_{i}\right)=0$ for all $m \geq x_{0}$. Again, by equation (13), this implies expected utility preferences. Similarly, by inspection of equation (13), $\sigma=0$ is identical to expected utility.

Property 6: Preferences in (3) are identical to Gul (1991) disappointment aversion when $\delta=1$ and $\sigma=1$.

Note that axiom 1 and 2 are identical to Gul and axioms 3 is identical to Gul's axioms when $\delta=1$. Finally, axiom 4 is satisfied only if $\sigma=1$ (or when preferences are expected utility).

Property 7: $\mu(p)$ defined by equation (3) is linearly homogeneous only if the Symmetry Axiom is satisfied (i.e., $\sigma=1$ ).

Consider two lotteries, $p$ and $p^{a}$ such that $p$ has probabilities $\left[p_{1}, p_{2}, \ldots\right]$ and payoffs $\left[x_{1}, x_{2}, \ldots\right]$ while $p^{a}$ has identical probabilities $\left[p_{1}, p_{2}, \ldots\right]$ but payoffs $\left[a x_{1}, a x_{2}, \ldots\right]$ for $a>0$. From equation (13), note that $M\left(p^{a}, a m\right)^{\alpha}=$ $[a M(p, m)]^{\alpha}$ for all $m$ only in the case where $\sigma=1$ or where preferences are identical to expected utility, that is, the Symmetry Axiom is satisfied. 
Asymmetric disappointment aversion may be an interesting direction for future research. Since it allows for a different degree of risk aversion over disappointing outcomes, it is similar to Chew (1989) weighted utility. It also has the potential to generate preferences that are analogous to the "S-shaped" valuation function in Prospect Theory (Kahneman and Tversky (1979)). Unfortunately, the lack of homothetheticity makes the preferences difficult to use in an asset pricing setting.

Consider the case where $\delta<1$ but $\sigma=1$. In this setting, there are several alternative ways to write the preferences. Equation (3) has a "penalty" for outcomes below the certainty equivalence. This is the format used in implementation of Gul disappointment aversion in Epstein and Zin (2001). When the penalty is linear $(\sigma=1)$, one can write the preferences in a similar fashion as used in Bekaert, Hodrick, and Marshall (1997) or Ang, Bekaert, and Liu (2002).

$$
u(\mu(p))=a^{-1}\left(E\left[u(x) 1_{\{x>\delta \mu(p)\}}\right]+(1+\beta) E\left[u(x) 1_{\{x \leq \delta \mu(p)\}}\right]\right),
$$

where $1_{\{\cdot\}}$ is an indicator function and

$$
a=\operatorname{Prob}\{x>\delta \mu(p)\}+\operatorname{Prob}\{x \leq \delta \mu(p)\}\left(1+\beta \delta^{\alpha}\right) .
$$

In this format, preferences are similar to expected utility where the probability of a disappointing outcome is re-weighted to capture the disappointment.

\subsection{Indifference Curves}

To understand the role of the axioms and resulting utility function, it is helpful to compare indifference curves in both the probability simplex and in statespace for expected utility, Gul disappointment aversion, and our Generalized Disappointment Aversion. Figure 1 shows a probability simplex over the payoffs $x_{1}<x_{2}<x_{3}$. In these examples, let $X=\left[x_{1}, x_{3}\right]$. Each line represents 
lotteries $p=\left[p\left(x_{1}\right), p\left(x_{2}\right), p\left(x_{3}\right)\right]^{\prime}$ that have the same utility, $\mu(p)=x$. Indifference curves are extended outside the simplex only to facilitate exposition and comparison. In Figure 1 preferences are expected utility $(\beta=0, \delta$ can be arbitrary). Notice that as implied by the independence axiom, the indifference curves are linear and parallel.

It is also helpful to consider indifference curves in state-space where probabilities of two states are fixed at $p\left(x_{1}\right)=p\left(x_{2}\right)=\frac{1}{2}$ and the lottery payoffs are varied. Figure 2 shows the familiar smooth trade-off between state 1 and state 2 payoffs implied by expected utility.

Figure 3 displays Gul Disappointment Aversion preferences $(\beta=2.0$, and, by definition, $\delta=1.0$ ). The axioms imply the "betweenness" property or that indifference sets are linear (i.e., $p \sim \lambda p+(1-\lambda) \mu(p))$. However, unlike expected utility in Figure 1, indifference curves are not parallel. The main structure of Disappointment Aversion preferences can be seen by considering the three indifference sets defined by $\mu(p)=x_{i}$ for $i=1,2,3$. (For comparison, these three indifference lines are highlighted in Figure 1 as well.) At $\mu(p)=$ 1.0, nothing is disappointing since this is the worst possible outcome and preferences are identical to expected utility. Next consider $\mu(p)=x_{2}$. The slope of the indifference curve is controlled by the parameter $\beta$. Since axiom 3 relaxes the independence axiom, the slope of $\mu(p)=x_{2}$ can differ from the slope of $\mu(p)=x_{1}$. Below the threshold $\mu(p)=x_{2}$ (lower right hand portion of Figure 3) only outcome $x_{1}$ is disappointing. Therefore, Axiom 3 places structure on preferences for lotteries $p_{1}$ and $p_{2}$ only if $p_{1}\left(x_{1}\right)=p_{2}\left(x_{1}\right)$ (lie on the same vertical plane). This produces the "fan" pattern in the lower right. Similarly, for lotteries above the $\mu(p)=x_{2}$ threshold, both $x_{1}$ and $x_{2}$ are disappointing. In this region, Axiom 3 influences preferences for lotteries if $p_{1}\left(x_{1}\right)+p_{1}\left(x_{2}\right)=p_{2}\left(x_{1}\right)+p_{2}\left(x_{2}\right)$ (lotteries lie on same horizontal plane). Again, this produces the "fan" pattern. Finally, Axiom 4 induces the symmetry between the two regions (reflected about the $\mu(p)=x_{2}$ line). For example, symmetry implies that $\mu(p)=x_{3}$, where every outcome is disappointing, is parallel to $\mu(p)=x_{1}$ and, hence, consistent with expected utility. 
Figure 4 shows the indifference curve for disappointment aversion in statespace. Since disappointment is defined relative to the certainty equivalent, the indifference curve has a kink at certainty. Below the forty-five degree line, it is the state two outcome (vertical axis) while above the forty-five degree line, the state one outcome is disappointing. The $\beta$ parameter controls how much extra weight the disappointing outcome receives and, hence, the sharpness of the kink.

Finally, Figure 5 shows the indifference lotteries for GDA $(\beta=2.0$, and $\delta=0.8)$ where utility is both "Gul-like" and "expected-utility-like." Again, there are three indifference curves to focus on, $\mu(p)=\frac{x_{i}}{\delta}$ for $i=1,2,3$. Here, the threshold certainty equivalents are all shifted by the preference parameter $\delta$. For certainty equivalents less than $\frac{x_{1}}{\delta}$, no outcomes are disappointing. For lotteries below $\mu(p)=\frac{x_{1}}{\delta}$, preferences are identical to expected utility and indifference curves are parallel. The indifference curve at $\mu(p)=\frac{x_{2}}{\delta}$ is the threshold where $x_{2}$ is disappointing. As in disappointment aversion, the parameter $\beta$ determines the slope of this indifference set. As with disappointment aversion in Figure 1, axiom 3 influences preferences differently above and below this threshold producing the "fanning" behavior. Finally, Axiom 4, the symmetry, acts in a similar manner to generate symmetric preferences about the $\mu(p)=\frac{x_{2}}{\delta}$ threshold. Note that fictitious lotteries with $\mu(p)=\frac{x_{3}}{\delta}$ (which is a certainty equivalent above the best outcome $x_{3}$ ), that have the property that all outcomes are disappointing which, by the symmetry axiom, is equivalent to expected utility and parallel to $\mu(p)=\frac{x_{1}}{\delta}$.

Figure 6 shows an indifference curve for GDA in state space. Here, disappointment occurs for outcomes less than $\delta=0.8$ times the certainty equivalence. For gambles close to certainty (shown as the center cone in Figure 6 ), preferences are identical to expected utility since neither outcome is disappointing. This is analogous to the lower right hand portion of Figure 5. Lotteries where state 2 payoff is very low (below the cone) are disappointing. Similarly for low state 1 payoffs. Note that the kink in the indifference curves is not at certainty. Here the kink occurs where the $x_{1}$ or $x_{2}$ is at the $\delta \mu(p)$ 
threshold. Finally, as long as $u(x)$ is homothetic, GDA preferences are also homothetic. For different utility levels, all kinks lie along the rays that define the cone in Figure 6.

Figures 2, 4 and 6 also show the role of $\beta$ and $\delta$ effect on effective risk aversion. In Disappointment Aversion, Figure 4, an increase in $\beta$ increases the severity of the kink and makes the preferences exhibit more risk aversion. This is also true in GDA in Figure 4. However, as $\delta$ decreases and the disappointment threshold is moved further away from certainty, the kink and effective risk aversion decreases. Monotonicity of preferences limits the kink since preferences can, at most, be a vertical (horizontal) line. When we turn to the empirical implementation of the model we will explore this tension further. Locating the kink away from certainty allows the calibration to locate the kink closer to the endowment. However, since the effect of $\beta$ is less dramatic, the effective disappointment aversion is reduced and preferences effectively look like expected utility.

\section{Intertemporal Asset Pricing with GDA}

We now embed our atemporal axiomatic preference model in a standard representative agent asset-pricing economy, as in Epstein and Zin (1990). The representative agent consumes a single perishable consumption good in each period. In period $t$, current consumption, $c_{t}$, is known with certainty, but future consumption levels are generally uncertain. The intertemporal utility functional is recursive and has the form

$$
U_{t}=\left[\left(1-\frac{1}{1+\rho}\right) c_{t}^{\gamma}+\frac{1}{1+\rho} \mu_{t}^{\gamma}\right]^{1 / \gamma}, \quad \gamma \leq 1, \quad \rho>0,
$$

where $\mu_{t}=\mu\left(\tilde{U}_{t+1} \mid I_{t}\right)$ as defined by equation (3) is the certainty equivalent of random future utility using the period- $t$ conditional probability distribution. $\rho$ is the marginal rate of time preference, and $\frac{1}{1-\gamma}$ is the elasticity of 
intertemporal substitution.

The representative agent's economic environment is identical to that in Mehra and Prescott (1985) and Melino and Yang (2002). In particular, the endowment process $\left\{y_{t}\right\}$ is such that growth rates $x_{t+1}=\frac{y_{t+1}}{y_{t}}$ follow a firstorder Markov process. The ex-dividend price of the single equity asset may be described by the time-invariant and positive function $p\left(x_{t}, y_{t}\right)$. In light of the homogeneity of preferences (Property 7 and equation (16)) it follows that price is linearly homogeneous in current output, i.e.,

$$
p(x, y)=p(x, 1) y=P(x) y,
$$

where $P(x)$ is the price-dividend ratio. Denote by $R_{t+1}$ the return to equity over the interval $t$ to $t+1$. This return depends only on the growth rate process and on price-dividend ratios via the equation:

$$
R_{t+1}=\frac{p\left(x_{t+1}, y_{t+1}\right)+y_{t+1}}{p\left(x_{t}, y_{t}\right)}=x_{t+1} \frac{\left(P\left(x_{t+1}\right)+1\right)}{P\left(x_{t}\right)} .
$$

In equilibrium, the agent maximizes utility, markets clear, and price expectations are fulfilled. The Euler equation for utility maximization from Epstein and Zin (1989) is given by

$$
\left[\mu_{t}\left(\tilde{x}_{t+1}^{(\gamma-1) / \gamma} \tilde{R}_{t+1}^{1 / \gamma}\right)\right]^{\gamma}=1+\rho
$$

If we substitute the definition of the market return from equation (18) into this Euler equation, and apply the property of linear homogeneity of the certainty equivalent operator, then we obtain a recursive equation for the equilibrium price function, as in Epstein and Zin (1990),

$$
\left.P\left(x_{t}\right)=\frac{1}{1+\rho}\left[\mu_{t}\left(\tilde{x}_{t+1}\left(P\left(\tilde{x}_{t+1}\right)+1\right)^{1 / \gamma}\right)\right)\right]^{\gamma} .
$$

The equilibrium risk-free return is determined by the representative agent's portfolio choice between the risky equity return $R_{t+1}$ and a risk-free asset with 
return $r_{t}$. From Epstein and Zin (1989), the individual's portfolio choice is determined by solving

$$
\max _{\omega} \mu_{t}\left(\left(\tilde{x}_{t+1} / \tilde{R}_{t+1}^{\omega}\right)^{(\gamma-1) / \gamma}\left(\omega \tilde{R}_{t+1}+(1-\omega) r_{t}\right)\right)
$$

where $\tilde{R}_{t+1}^{\omega}=\omega \tilde{R}_{t+1}+(1-\omega) r_{t}$ is the portfolio return. The equilibrium riskfree return, occurs when the optimum of this portfolio choice problem is $\omega=1$. That is, the risk-free asset is priced such that asset demands are consistent with zero net supply.

Of particular interest for understanding some of the simple calibration exercises below, will be the version of our model that is linear-in-states. When $\gamma=1$, intertemporal substitution is perfectly elastic and (21) reduces to

$$
\max _{\omega} \mu_{t}\left(\omega \tilde{R}_{t+1}+(1-\omega) r_{t}\right)
$$

Consider an outcome-valuation function that is homogeneous of degree one (i.e., $\alpha=1$ in equation (3)). Given the linear homogeneity of $\mu$, equation (21) reduces further to

$$
\max _{\omega}\left[\omega \mu_{t}\left(\tilde{R}_{t+1}\right)+(1-\omega) r_{t}\right],
$$

Note that in this special case the certainty equivalent is a linear operator. This implies that, in equilibrium,

$$
\mu_{t}\left(\tilde{R}_{t+1}\right)=r_{t}
$$

From equation (20) with $\gamma=1$

$$
\mu_{t}\left(\tilde{R}_{t+1}\right)=1+\rho
$$

Therefore, in the equilibrium

$$
\mu_{t}\left(\tilde{R}_{t+1}\right)=r_{t}=1+\rho
$$

Note that this linear specification with $\alpha=1$ and $\gamma=1$ represents risk- 
neutrality only in the expected utility model. For generalized disappointment aversion models with $\beta>0$, (see equation (3)), the certainty equivalent will exhibit risk aversion.

\subsection{Calibration}

To investigate the quantitative implications of our model of risk preferences, we adopt the two-state calibration of the endowment-growth process described in Mehra and Prescott (1985). As described in the introduction, the expected value of and standard deviation of the endowment growth rate are 1.018 and 0.036, respectively. For a symmetric ergodic distribution, i.e., an unconditional probability of 0.5 , the two growth-rate states are $x^{L}=1.018-0.036=0.982$ and $x^{H}=1.018+0.036=1.054$. The first-order autocorrelation of these growth rates is calibrated to be -0.14 , which implies the Markov transition matrix shown in equation (1).

Given this specification for the exogenous endowment process and given values for the parameters of the utility function, we can solve the equations described above to determine the equilibrium asset returns. Given these returns, we can solve for the 2-by-2 matrix defining the equilibrium asset-pricing kernel, $M$, that satisfies the 4 equations

$$
\begin{aligned}
M_{L L} \pi_{L L} R_{L L}+M_{L H} \pi_{L H} R_{L H} & =1 \\
M_{H L} \pi_{H L} R_{H L}+M_{H H} \pi_{H H} R_{H H} & =1 \\
M_{L L} \pi_{L L} r_{L}+M_{L H} \pi_{L H} r_{L} & =1 \\
M_{H L} \pi_{H L} r_{H}+M_{H H} \pi_{H H} r_{H} & =1 .
\end{aligned}
$$

Our calibration approach is somewhat non-standard. We choose preference parameters for each preference model so that the equilibrium asset-pricing kernel, $M$, is as close (in a sum-of-squared-errors sense) as possible to the matrix found by Melino and Yang (2002). That is, we ask how close can a particular preference model get the the Melino-Yang pricing kernel given in 
equation (2). We have not tried to pre-specify preference parameters from other empirical or experimental evidence. Of course, such evidence will be relevant for evaluating the results of our numerical exercise.

The results of this calibration, along with the preference parameters, are presented in Table 1. The table reports six preference models: time-additive expected utility as in Mehra and Prescott (1985) (denoted EU), Kreps and Porteus (1978) non-time-additive expected utility as in Epstein and Zin (1989) (denoted KP), the Disappointment Aversion model of Gul (1991) (denoted DA), Disappointment Aversion utility constrained to be linear in states (i.e., $\alpha$ is constrained to be equal to 1) (denoted DAL), Generalized Disappointment Aversion (GDA), and finally Generalized Disappointment Aversion with the constraint $\alpha=1$ (GDAL). For all models, the rate of time-preference is fixed at $\rho=0.01$.

Despite the variety of preferences considered, all six models presented in Table 1 generate roughly the same pricing kernels. None of these pricing kernels exhibits the big differences in conditional behavior depicted in Melino and Yang (2002). In other words, although each model has the potential to generate different risk premiums, different risk-free interest rates and different volatility patterns in these returns, none of the models comes close to resolving the equity-premium puzzle. This is true even when the parameters of the model are chosen with that specific goal in mind. Finally, note that the curvature of $u$ in the certainty equivalents for both disappointment aversion and generalized disappointment aversion does not have much of a quantitative impact in this two-state economy. Almost identical results obtain when a $\alpha=1$ and a linear-in-states value function is used. This is, of course, not surprising since both $\alpha$ and $\beta$ induce risk aversion (see Property 3 or Figures 4 and 6).

\subsection{Discussion}

To understand where the GDA utility calibration may be useful, it is helpful to consider a simpler two-period two-state model. In this setting, consump- 
tion is denoted $c_{1}$ and $c_{2}$. Today's endowment, $y_{1}$ is known. The growth rate for tomorrow's endowment can be $x_{L}$ or $x_{H}$, which along with the consumption/savings plan will produce period-two consumption of $c_{2}$. Figure 7 characterizes the representative agent's problem. The lower left quadrant shows preferences over risky consumption growth rates $\left(x_{L}, x_{H}\right)$. The upper right quadrant shows the intertemporal preference between certain (or certainty equivalent) consumption plans $\left(c_{1}, \mu\left(c_{2}\right)\right)$. The endowment is $\left(y_{1}, \bar{x}_{L}, \bar{x}_{H}\right)$. In Figure 7, preferences over atemporal gambles (lower-left quadrant) are standard expected utility. Equilibrium is given by setting state prices $\frac{p_{H}}{p_{L}}$ equal to the slope of the indifference curve at the endowment growth rates $\left(\bar{x}_{L}, \bar{x}_{H}\right)$. Where the indifference curve that runs through $\left(\bar{x}_{L}, \bar{x}_{H}\right)$ intersects certainty (where $x_{L}=x_{H}$ ) determines the certainty equivalence of the period-two risky endowment. This is denoted $\mu(\bar{x})$. The upper-left quadrant simply maps the certainty equivalence of risky growth-rates into period 2 consumption certainty equivalence. Since our certainty equivalence are linearly homogeneous, $\mu\left(y_{2}\right)=y_{1} \mu(\tilde{x})$ where $\tilde{x}$ is the $\left(x_{L}, x_{H}\right)$ gamble. The equilibrium interest rate is therefore set by the slope of the intertemporal trade-off at $\left(y_{1}, \mu\left(y_{2}\right)\right)$.

From Figure 7, one can see why the expected utility model struggles to match the data. Consider the comparative static exercise of changing the probabilities of the two states in a way analogous to the two-state infinite horizon probabilities in Melino and Yang (2002). That is, consider $\pi_{H} / \pi_{L}=$ 0.75 or 1.33 (see equation (1)). Regardless of the risk aversion curvature, one cannot duplicate the Melino and Yang (2002) state prices since small change in probabilities cannot reproduce the dramatic variation in conditional state prices. Note even with state-dependent risk aversion, that allows one to match the conditional state prices (the $p_{H} / p_{L}$ in the lower-left quadrant of the figure), expected utility will produce a dramatic variation in the certainty equivalents of the consumption growth. This translates into an interest rate that is excessively volatile.

In contrast, Figure 8 shows the two-period economy with a representative agent with GDA utility over growth rates. By choice of the parameters $\delta$, 
the kink in the utility function can be located closer to the endowment. The effect is to separate the slope of the utility function at $\left(\bar{x}_{L}, \bar{x}_{H}\right)$ (determining state prices $\left.\frac{p_{H}}{p_{L}}\right)$ from the certainty equivalence that determines the interest rate. If we consider the same comparative static exercise as above (consider $\pi_{H} / \pi_{L}=0.75$ or 1.33$)$, the $\beta$ parameter allows one to overweight the disappointing outcomes and effectively exaggerate the small probability change into a larger effect on the state prices. Since the kink is near the endowment, this exaggeration of state prices is done without generating much variation in the certainty equivalence and hence without inducing a volatile risk-free rate. However, as noted earlier, the continuity and monotonicity restrictions on preferences limit the amount of the kink. As such, it is hard to generate a $\beta$ that is large enough to reproduce the Melino and Yang (2002) state prices. This is seen in Table 1. None of the preference models can reproduce the Melino-Yang prices. Ideally, what we need is for the endowment to lie on either side of the disappointment threshold in a state-dependent way. While this is hard to demonstrate in a picture (since the endowment growth rates is fixed), we explore if this is feasible in the in the infinite horizon case below. As is shown next, Generalized Disappointment Aversion can generate the effective state-dependent risk aversion if the endowment growth process has a small degree of heteroskedasticity.

\subsection{Calibration with Conditional Volatility}

Building on the intuition from the previous discussion, we consider a slightly different calibration of the exogenous endowment-growth process than in Mehra and Prescott (1985). Although it seems relatively innocuous to assume a symmetric ergodic distribution $(p=1 / 2)$, this assumption rules out conditional heteroskedasticity the in two-state models. Since there is ample evidence from both macroeconomic and financial-markets data that indicates conditional volatility as an empirical fact, it is worth exploring in greater detail. In particular, the emphasis Generalized Disappointment Aversion places on 
extreme tail events suggests that conditional heteroskedasticity may be important to distinguish GDA preferences from other preference models in a dynamic economy.

The conditional means of the endowment growth process in the 2-state model are given by

$$
\begin{aligned}
E\left(x_{t+1} \mid x_{t}=x_{L}\right) & =\mu_{x}-\theta\left(\frac{1-p}{p}\right)^{1 / 2} \sigma_{x} \\
& =(1-\theta) \mu_{x}+\theta x_{L}
\end{aligned}
$$

and

$$
\begin{aligned}
E\left(x_{t+1} \mid x_{t}=x_{H}\right) & =\mu_{x}+\theta\left(\frac{p}{1-p}\right)^{1 / 2} \sigma_{x} \\
& =(1-\theta) \mu_{x}+\theta x_{H}
\end{aligned}
$$

where $\mu_{x}$ and $\sigma_{x}$ are the unconditional (ergodic) mean and standard deviation, $p$ is the ergodic probability of $x=x_{L}$, and $\theta$ is the first-order autocorrelation coefficient.

The conditional variances are given by

$$
V\left(x_{t+1} \mid x_{t}=x_{L}\right)=\sigma_{x}^{2}\left[1+\theta \frac{1-2 p}{p}-\theta^{2} \frac{1-p}{p}\right]
$$

and

$$
V\left(x_{t+1} \mid x_{t}=x_{H}\right)=\sigma_{x}^{2}\left[1-\theta \frac{1-2 p}{1-p}-\theta^{2} \frac{p}{1-p}\right]
$$

Note that the difference in conditional variances is given by

$$
V\left(x_{t+1} \mid x_{t}=x_{L}\right)-V\left(x_{t+1} \mid x_{t}=x_{H}\right)=2 \theta \frac{1-2 p}{p(1-p)}
$$

As noted, the only case in which there is no conditional heteroskedasticity in the 2-state model is precisely the Mehra-Prescott calibration with equal ergodic probability on the two states $(p=.5)$. 
Consider two relatively small changes to the Melino-Yang exercise. First, we introduce a small amount of conditional heteroskedasticity into the endowmentgrowth process by assuming an ergodic probability of the low growth state of $p=0.4$. This implies transition probabilities

$$
\left[\begin{array}{cc}
\pi_{L L} & \pi_{L H} \\
\pi_{H L} & \pi_{H H}
\end{array}\right]=\left[\begin{array}{ll}
0.316 & 0.684 \\
0.456 & 0.544
\end{array}\right]
$$

Note the conditional standard deviation in the low state is 0.0342 , and in the high state it is 0.0366 . Given the unconditional standard deviation is equal to 0.036 , this is a reasonably small amount of conditional heteroskedasticity. Second, we set the risk-free interest rate to a constant equal to the rate of time preference, $r_{t}=1.01$ for all $t$. The average equity premium we try to match is 0.08 , a slight increase over Mehra-Prescott. Re-solving the equations in Melino and Yang (2002) with these assumptions results in a pricing kernel given by

$$
\left[\begin{array}{ll}
M_{L L} & M_{L H} \\
M_{H L} & M_{H H}
\end{array}\right]=\left[\begin{array}{cc}
2.4811 & 0.3013 \\
0.9914 & 0.9890
\end{array}\right]
$$

This pricing kernel exhibits that same patterns as the Melino-Yang matrix in equation (2) and is an equally challenging test for asset-pricing theories. Table 2 displays results for this calibration. What is striking about the results in Table 2 is the perfect fit of the generalized disappointment aversion preferences. This fit occurs even though we have fixed the curvature parameter in the $u$ of equation (3) at the value $\alpha=1$.

To understand this rather surprising result, consider equation (22) and the gambles implied by an ownership claim to equity. The matrix of equity returns consistent with equilibrium asset prices in this particular calibration is given by

$$
\left[\begin{array}{ll}
R_{L L} & R_{L H} \\
R_{H L} & R_{H H}
\end{array}\right]=\left[\begin{array}{cc}
0.9227 & 1.3420 \\
0.9400 & 1.0688
\end{array}\right] .
$$


whereas in the Mehra-Prescott calibration this matrix is given by

$$
\left[\begin{array}{ll}
R_{L L} & R_{L H} \\
R_{H L} & R_{H H}
\end{array}\right]=\left[\begin{array}{ll}
1.0238 & 1.2953 \\
0.8631 & 1.0919
\end{array}\right]
$$

The important difference in these two matrices lies in comparing the smallest value in each row. In the Mehra-Prescott economy in equation (27), the smallest return in the first row, 1.0238 in event $(L, L)$, is larger than the smallest value in the second row, 0.8631. In our calibration with conditional heteroskedasticity in equation (26), the smallest value in the first row, 0.9227 is smaller than the smallest element in the second row, 0.9400. The reason this is important is that Generalized Disappointment Aversion preferences may allow the conditional outcomes in the second row (expansion state) to not be disappointing while outcomes in the first row (recession state) are potentially disappointing. That is, there is a value of $\delta$ such that $\delta \mu_{t}<0.9400$ when $x_{t}=x_{H}$ but have $\delta \mu_{t}>0.9227$ when $x_{t}=x_{L}$. In this situation, the investor will behave in a risk-neutral fashion when they are in the "high" state, and a very risk-averse fashion when they are in the "low" state. GDA effectively creates state-dependent risk aversion. Note that this is not possible for MehraPrescott calibration in equation (27). In addition, effective state dependent risk aversion is not possible if $\delta=1$. Standard disappointment aversion cannot reproduce the dynamic features of asset prices. Beyond this two-state calibration, this type of state-dependent risk aversion will arise whenever there are significantly more lower-tail events in one conditional probability distribution than in another, hence, the important role played by conditional heteroskedasticity in our calibration.

\section{Conclusion}

We have provided an axiomatic generalization of the disappointment aversion preferences of Gul (1991) which allow for a more flexible definition of a "disappointing" outcome. In particular, our one-parameter extension of Gul's utility 
function allows us to characterize outcomes in a lottery as disappointing not when they lie below that lottery's certainty equivalent, but rather when they are sufficiently far below the certainty equivalent. This focus on more extreme tail behavior is consistent with real-world approaches such as "Value at Risk" calculations in finance (see Basak and Shapiro (2001)), and with much of the behavioral/experimental evidence, such as Kahneman and Tversky (1979). Like Gul's approach, our generalization maintains the key assumptions of linearity of indifference curves on the probability simplex, linear homogeneity of certainty equivalents, monotonicity (first-order stochastic dominance), and risk aversion (second-order stochastic dominance), while allowing for departures from the independence axiom of expected utility. These features were shown in Epstein and Zin (2001) to play a key role in formulating tractable dynamic asset-pricing models that allow for risk-preferences that depart from expected utility in empirically relevant ways.

When we embed our new model of risk preferences in a Lucas-tree assetpricing economy, we find that its quantitative importance is linked to the existence of a small amount of conditional heteroskedasticity in the 2-state Markov process for the endowment growth. If homoskedasticity is assumed as in Mehra and Prescott (1985), our model offers little improvement over existing models. On the other hand, in the presence of a relatively small amount of heteroskedasticity, our model generates state-prices that are consistent with the historical patterns of equity and bond returns as delineated in Mehra and Prescott (1985). In particular, our preference specification generates effective risk aversion that is both state-dependent and counter-cyclical. The empirical finance literature has highlighted the importance of these two properties as necessary features of any asset pricing model that is likely to fit the data. Moreover, our axiomatic approach allows us to have a deeper understanding of the origins of this time-varying risk aversion and allows us to better evaluate the structural stability of our preference parameters.

Future research will take further advantage of the tractability of the Euler equations in our model to explore the empirical implications of our new 
preference specification using both Hansen-Jagannathan bounds and GMM estimation as in Epstein and Zin (2001) and general-equilibrium term-structure puzzles as in Backus, Gregory, and Zin (1989). In addition, we will explore the usefulness of our model for understanding partial-equilibrium portfolio-choices. 


\section{References}

Allais, M. (1979): "The Foundations of a Positive Theory of Choice Involving Risk and a Criticism of the Postulates and Axioms of the American Schhol," in Expected Utility Hypothesis and the Allais Paradox, ed. by M. Allais, and O. Hagen. D. Reidel Publishing Co., Dordrecht, Holland.

Ang, A., G. Bekaert, and J. LiU (2002): "Why Stocks May Disappoint," NBER Working Paper w7783, Working Paper.

Backus, D. K., A. W. Gregory, and S. E. Zin (1989): "Risk Premiums in the Term Structure: Evidence from Artificial Economies.," Journal of Monetary Economics, 24, 371-399.

Barberis, N., and M. Huang (2001): "Mental Accounting, Loss Aversion, and Individual Stock Returns," The Journal of Finance, LVI(4), 1247.

Barberis, N., M. Huang, and T. Santos (2001): "Prospect Theory and Asset Prices," The Quarterly Journal of Economics, CXVI(1).

Basak, S., And A. Shapiro (2001): "Value-at-Risk Based Risk Management: Optimal Policies and Asset Prices," Review of Financial Studies, 14, 371-405(2), 371-405.

Bekaert, G., R. J. Hodrick, and D. A. Marshall (1997): "The implications of first-order risk aversion for asset market risk premiums," Journal of Monetary Economics, 40(1), 3-39.

Campbell, J. Y., And J. H. Cochrane (1999): "By Force of Habit: A Consumption-Based Explanation of Aggregate Stock Market Behavior," Journal of Political Economy, 107, 205-251.

Chew, S. H. (1989): "Axiomatic Utility Theories with the Betweeness Property," Annals of Operations Research, 19, 273-298.

Conlisk, J. (1989): "Three Variants on the Allais Paradox," The American Economic Review, 79(3), 392-407.

Dekel, E. (1986): "An Axiomatic Characterization of Preferences under Uncertainty," Journal of Economic Theory, 40, 304-318.

Epstein, L., And S. E. ZIN (2001): "The independence axiom and asset returns," Journal of Empirical Finance, 8, 537-572. 
Epstein, L. G., And S. E. Zin (1989): "Substitution, Risk Aversion, and the Temporal Behavior of Consumption and Asset Returns: A Theoretical Framework," Econometrica, 57(4), 937-969.

_ (1990): "First-Order" Risk Aversion and the Equity Premium Puzzle," Journal of Monetary Economics, 26(3), 387-407.

Gordon, S., And P. St-Amour (2000): "A Preference Regiem Model of Null and Bear Markets," American Economic Review, 90(4), 1019-1033.

Gul, F. (1991): "A Theory of Disappointment Aversion," Econometrica, $59(3), 667-686$.

Kahneman, D., And A. Tversky (1979): "Prospect Theory: An Analysis of Decision under Risk," Econometrica, 47(2), 263-292.

Kreps, D. M., and E. L. Porteus (1978): "Temporal Resolution of Uncertainty," Econometrica, 46, 185-200.

LucAs, JR., R. E. (1978): "Asset Prices in an Exchange Economy," Econometrica, 46, 1426-14.

Machina, M. J. (1987): "Choince Under Uncertainty: Problems Solved and Unsolved," Journal of Economic Perspectives, 1(1), 121-154.

Mehra, R., and E. Prescott (1985): "The Equity Premium: A Puzzle," Journal of Monetary Economics, 15, 145-161.

Melino, A., And A. X. YAng (2002): "State Dependent Preferences Can Explain the Equity Premium Puzzle," University of Toronto Working Paper.

SAGi, J. S. (2003): "Anchored Preference Relations," Working Paper, University of California at Berkeley.

von Neumann, J., and O. Morgenstern (1947): Theory of Games and Economic Behavior. Princeton University Press, Princeton, NJ. 


\section{Appendix}

Lemma: Preferences satisfy axioms 0,1,2, and "betweenness," if and only if there exists

(i) $u: X \rightarrow[0,1] \quad$ continuous, increasing

(ii) $\Delta_{k}: X \rightarrow X \quad$ continuous, increasing

$\Delta_{k}(m)<\Delta_{k+1}(m)$ for all $m \in X, k=1, \ldots, K-1$, and $\Delta_{K}(m) \geq x^{0}$ for all $m$

(iii) $\quad L_{k}(x, m):[0,1]^{2} \rightarrow \mathbb{R}$ continuous, decreasing in $x$, increasing in $m$

(iv) $\beta_{k} \in(-1, \infty)$

and

$$
M(p, m)=u^{-1}\left(\sum_{x_{i} \in X} p\left(x_{i}\right) u\left(x_{i}\right)-\sum_{k=1}^{K} \beta_{k} \sum_{x_{i} \leq \Delta_{k}(m)} p\left(x_{i}\right) L_{k}\left(x_{i}, \Delta_{k}(m)\right)\right)
$$

such that $\mu(p)=M(p, \mu(p))$ and $p \succeq q$ if and only if $\mu(p) \geq \mu(q)$.

Proof: Dekel (1986) and Chew (1989) show that preferences satisfy axioms 0,1,2, and betweenness if and only if there exists a function $U: \mathcal{L} \times X \rightarrow \mathbb{R}$ such that $\mu(p)$ uniquely solves

$$
\sum_{x_{i} \in X} p\left(x_{i}\right) U\left(x_{i}, \mu(p)\right)=0
$$

with $U(x, m)$ continuous, increasing in $x$, decreasing in $m$ and satisfies $U(x, m)=0$ if $x=m$ (and with the normalization that $\mu\left(x_{0}\right)=0$ and $\mu\left(x^{0}\right)=1$ ). Converting (A2) to (A1) is simply notation and algebra. Rewrite (A2) as

$$
\sum_{x_{i} \in X} p\left(x_{i}\right) u\left(x_{i}\right)-\sum_{x_{i} \in X} p\left(x_{i}\right)\left(U\left(x_{i}, m\right)+u\left(x_{i}\right)-u(m)\right)=u(m)
$$

Let $F(x, m)=U(x, m)+u(x)-u(m)$. Note $F(x, m)$ is continuous and increasing in $x$, decreasing in $m$, and $F(x, m)=0$ if $x=m$. Since the function is continuous, we can now partition this function (note $\Delta_{k}(\cdot)$ are invertible). Let

$$
\begin{aligned}
& L_{1}(x, z)=F\left(x, \Delta_{k}^{-1}(z)\right) 1_{\{x \leq z\}} \\
& L_{k}(x, z)=F\left(x, \Delta_{k}^{-1}(z)\right) 1_{\{x \leq z\}}-\sum_{h=1}^{k-1} L_{k}(x, z) \quad k=2, \ldots, K
\end{aligned}
$$

Let $z=\Delta_{k}(m)$ and note $L\left(x, \Delta_{k}(m)\right)=0$ at $x=\Delta_{k}(m)$. Finally, rescale the function by $\beta_{k}$. 
Table 1: Mehra-Prescott Calibration Results Matching Mehra-Prescott/Melino-Yang State Prices

\begin{tabular}{crrrrrrr}
\hline \hline & & \multicolumn{7}{c}{ Risk-Preference Model } \\
Parameter & Yang & EU & KP & DA & DAL & GDA & GDAL \\
\cline { 3 - 8 }$\alpha$ & & & & & & & \\
$\gamma$ & & -12.65 & -10.95 & -2.10 & 1.00 & -1.95 & 1.00 \\
$\beta$ & & $1 / \alpha$ & -1.26 & -1.51 & -1.15 & -1.52 & -1.20 \\
$\delta$ & & $0^{*}$ & $0^{*}$ & 1.15 & 1.75 & 1.25 & 1.70 \\
& & $n a *$ & $n a *$ & $1^{*}$ & $1^{*}$ & 0.98 & 0.995 \\
& & & & & & & \\
$M_{L L}$ & 1.8620 & 1.5664 & 1.5812 & 1.5542 & 1.5241 & 1.5462 & 1.4992 \\
$M_{L H}$ & 0.2440 & 0.5284 & 0.4899 & 0.5372 & 0.5665 & 0.5265 & 0.5788 \\
$M_{H L}$ & 1.1270 & 1.3733 & 1.3889 & 1.3829 & 1.3502 & 1.3708 & 1.3455 \\
$M_{H H}$ & 0.9490 & 0.4654 & 0.4323 & 0.4796 & 0.5231 & 0.4680 & 0.5222 \\
& & & & & & & \\
\hline & & & & & & & \\
$E(R)$ & 1.070 & 1.040 & 1.055 & 1.036 & 1.032 & 1.047 & 1.035 \\
$\operatorname{std}(R)$ & 0.165 & 0.037 & 0.044 & 0.044 & 0.042 & 0.043 & 0.042 \\
$E(r)$ & 1.008 & 1.022 & 1.033 & 1.016 & 1.014 & 1.027 & 1.017 \\
$\operatorname{std}(r)$ & 0.056 & 0.004 & 0.010 & 0.010 & 0.008 & 0.009 & 0.009 \\
& & & & & & & \\
\hline
\end{tabular}

The Melino-Yang pricing kernel is from equation (2). Six different models are presented: $\mathrm{EU}$ is time-additive expected utility, $\mathrm{KP}$ is Kreps-Porteus recursive non-time-additive expected utility for atemporal gambles, DA is Recursive Disappointment Aversion utility, DAL is Recursive Disappointment Aversion utility with risk-aversion parameter $\alpha=1(u(x)$ linear $)$, GDA is Recursive Generalized Disappointment Aversion, and GDAL is Recursive Generalized Disappointment Aversion with risk-aversion parameter $\alpha=1(u(x)$ linear $)$. $\alpha$ is the riskaversion in $u(x) ; \frac{1}{1-\gamma}$ is the intertemporal elasticity; $\beta$ is the coefficient of loss-aversion (see equation $(3)) ; \delta$ is the threshold for disappointment (see equation (3)) For all models, the rate of time-preference is constrained to be $\rho=0.01$.

* $\beta=0$ implies expected utility and $\delta$ is undefined;

** $\delta=1.0$ implies Disappointment Aversion 
Table 2: Alternative Calibration Conditional Heteroskedasticity State Prices

\begin{tabular}{crrrr} 
& & \multicolumn{3}{c}{ Risk-Preference Model } \\
\cline { 3 - 5 } Parameter & $p=0.4$ & KP & DAL & GDAL \\
\hline & & & & \\
$\gamma$ & & -12.02 & 1.00 & 1.00 \\
$\beta$ & & -2.10 & -1.09 & 1.00 \\
$\delta$ & & $0^{*}$ & 1.93 & 1.61 \\
& & $n a^{*}$ & $1^{*}$ & 0.93 \\
& & & & \\
$M_{L L}$ & 2.4812 & 2.0733 & 1.9345 & 2.4812 \\
$M_{L H}$ & 0.3013 & 0.4499 & 0.5454 & 0.3013 \\
$M_{H L}$ & 0.9914 & 1.0534 & 1.6469 & 0.9914 \\
$M_{H H}$ & 0.9890 & 0.3755 & 0.4655 & 0.9890 \\
& & & & \\
\hline & & & & \\
$E(R)$ & 1.090 & 1.053 & 1.029 & 1.090 \\
$s t d(R)$ & 0.165 & 0.049 & 0.042 & 0.165 \\
$E(r)$ & 1.010 & 1.020 & 1.004 & 1.010 \\
$s t d(r)$ & 0.000 & 0.015 & 0.010 & 0.000 \\
& & & & \\
\hline \hline
\end{tabular}

The pricing kernel for the $p=0.4$ calibration is shown in (25) and (24). Three different models are presented: KP is Kreps-Porteus recursive non-time-additive expected utility for atemporal gambles, DAL is Recursive Disappointment Aversion utility with risk-aversion parameter $\alpha=1(u(x)$ linear), and GDAL is Recursive Generalized Disappointment Aversion with risk-aversion parameter $\alpha=1(u(x)$ linear $)$. $\alpha$ is the risk-aversion in $u(x) ; \frac{1}{1-\gamma}$ is the intertemporal elasticity; $\beta$ is the coefficient of loss-aversion (see equation $(3)) ; \delta$ is the threshold for disappointment (see equation (3)) For all models, the rate of timepreference is constrained to be $\rho=0.01$.

* $\beta=0$ implies expected utility and $\delta$ is undefined;

** $\delta=1.0$ implies Disappointment Aversion 
Figure 1: Indifference Simplex - Expected Utility

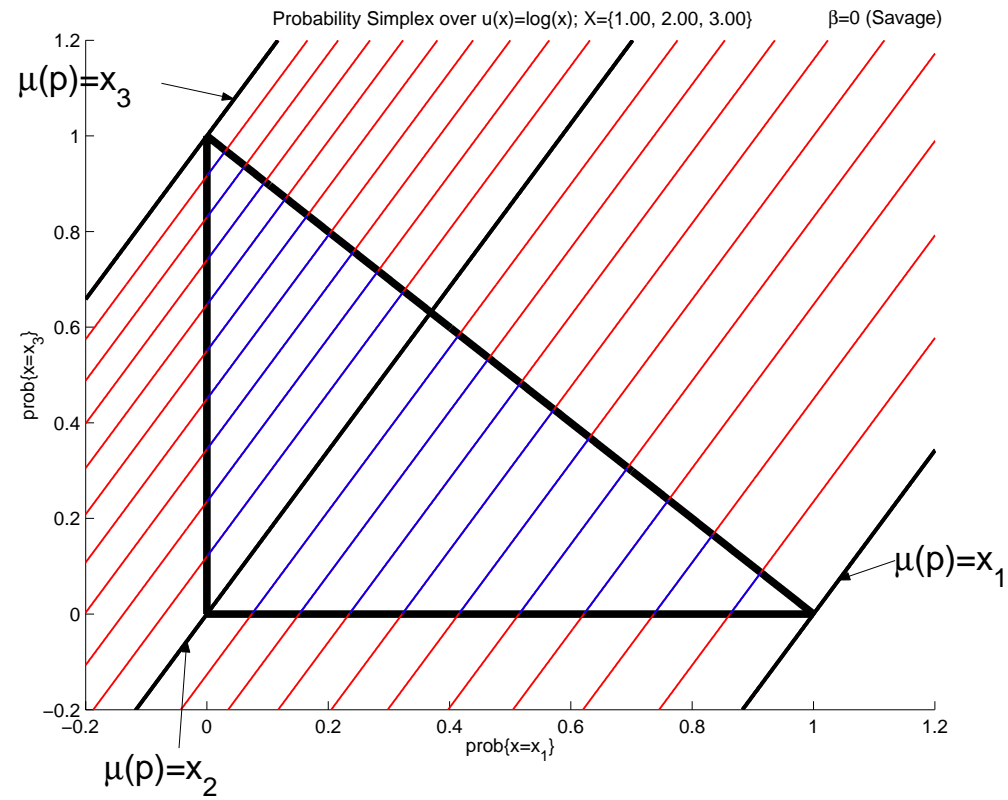

Probability simplex over payoffs $x_{1}=1, x_{2}=2$, and $x_{3}=3$ with value function $u(x)=\ln (x)$.

Figure 2: Indifference State-Space- Expected Utility

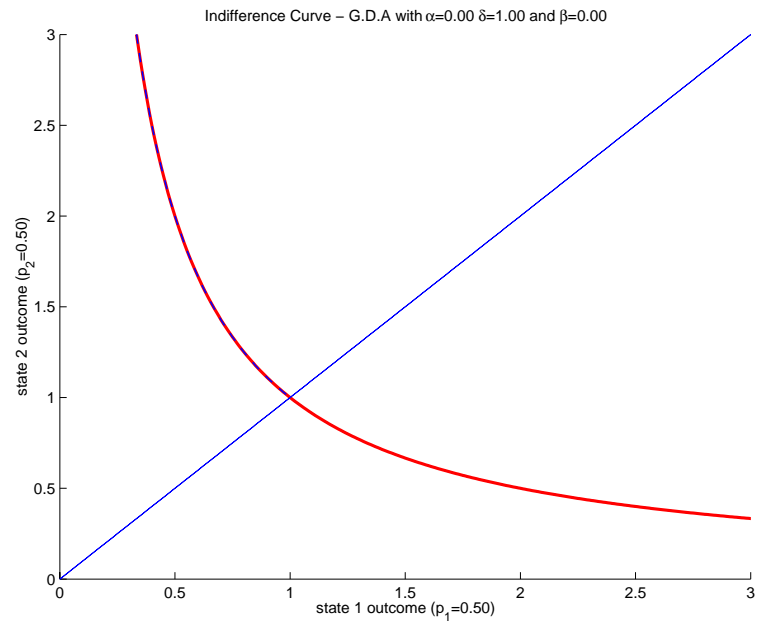

Two states have equal probabilities, $p\left(x_{1}\right)=p\left(x_{2}\right)=\frac{1}{2}$ with value function $u(x)=\ln (x)$. 
Figure 3: Indifference Simplex - Gul Disappointment Aversion

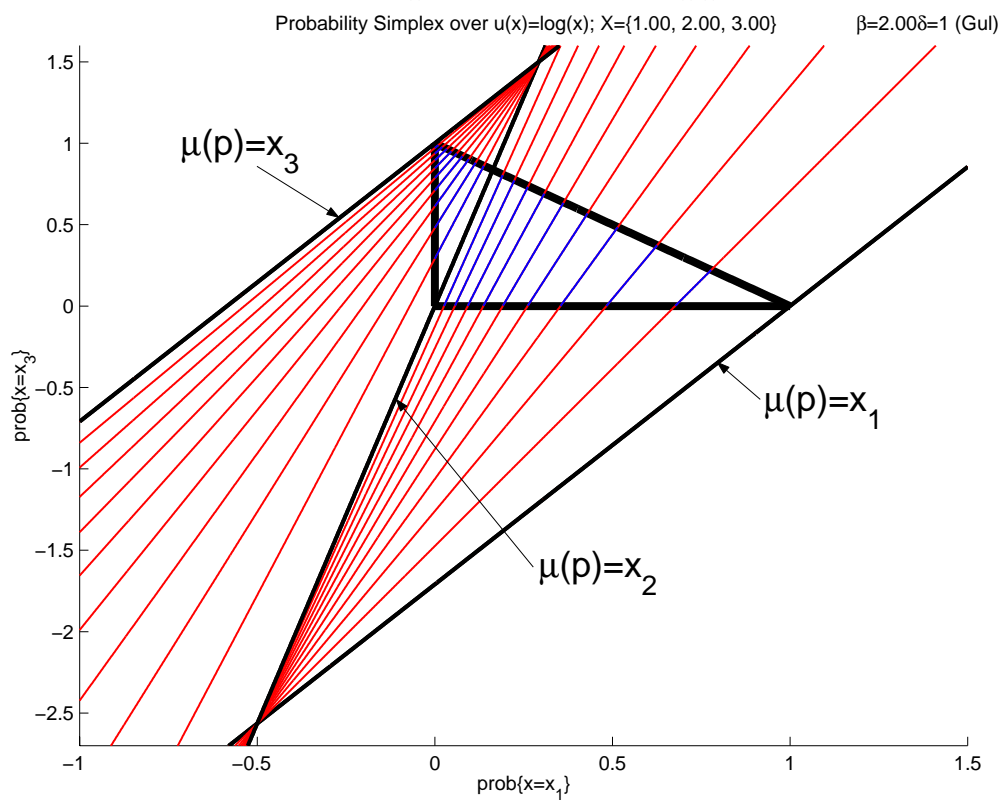

Description Here

Figure 4: Indifference Curve: Gul Disappointment Aversion

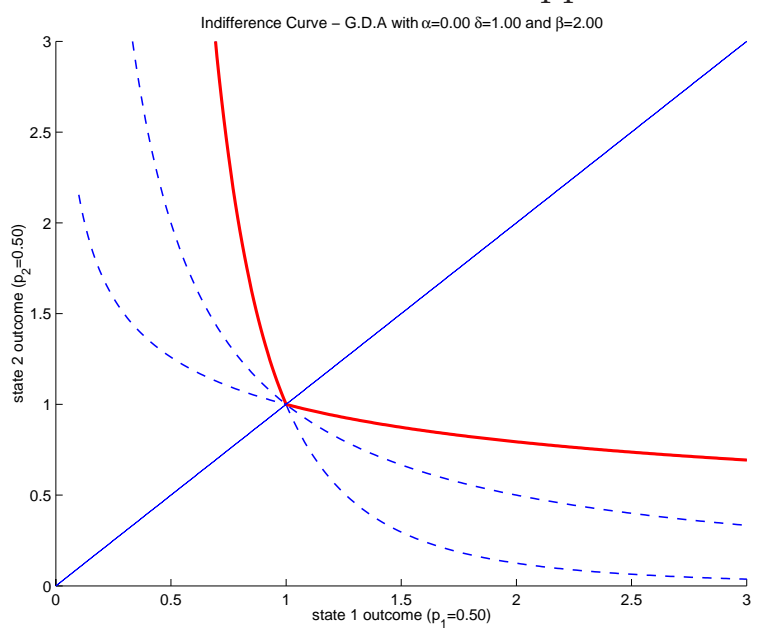

Description Here 
Figure 5: Indifference Simplex - GDA

Probability Simplex over $\mathrm{u}(\mathrm{x})=\log (\mathrm{x}) ; \mathrm{X}=\{1.00,2.00,3.00\}$ $\beta=2.00 \delta=0.80,(G D A)$

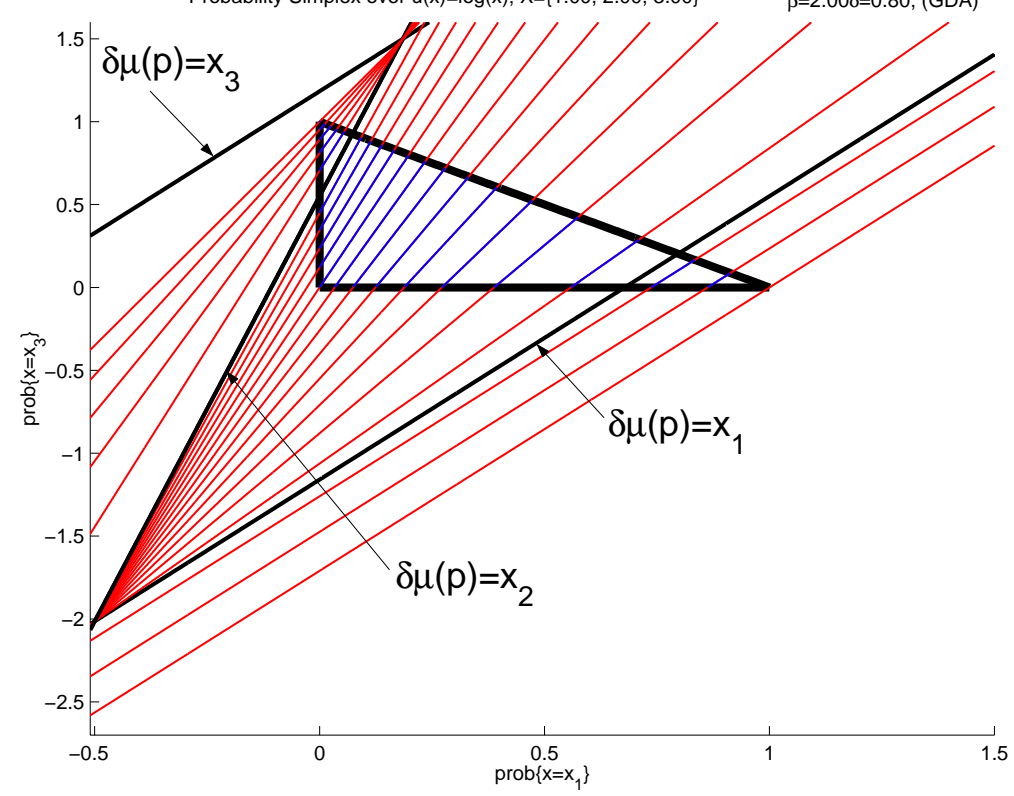

Description Here

Figure 6: Indifference Curve: Generalized Disappointment Aversion

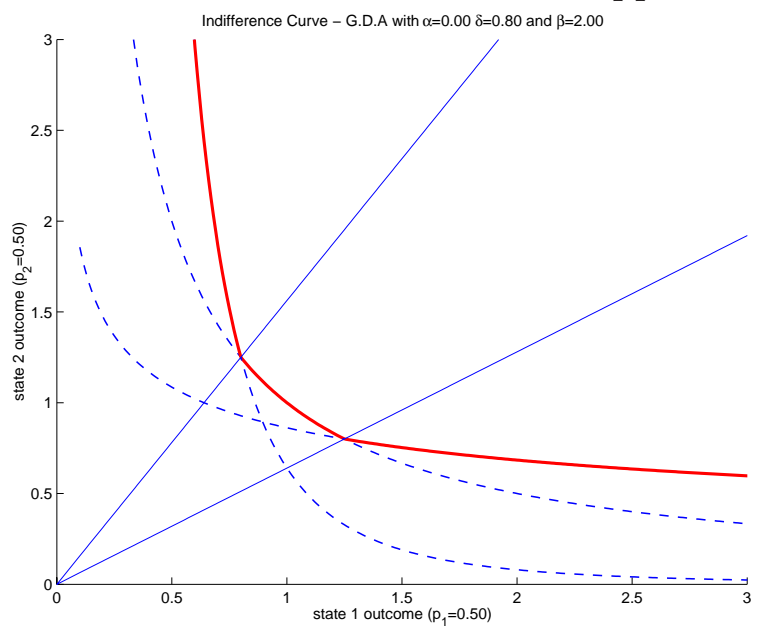

Description Here 
Figure 7: Two-Period Equilibrium - Expected Utility

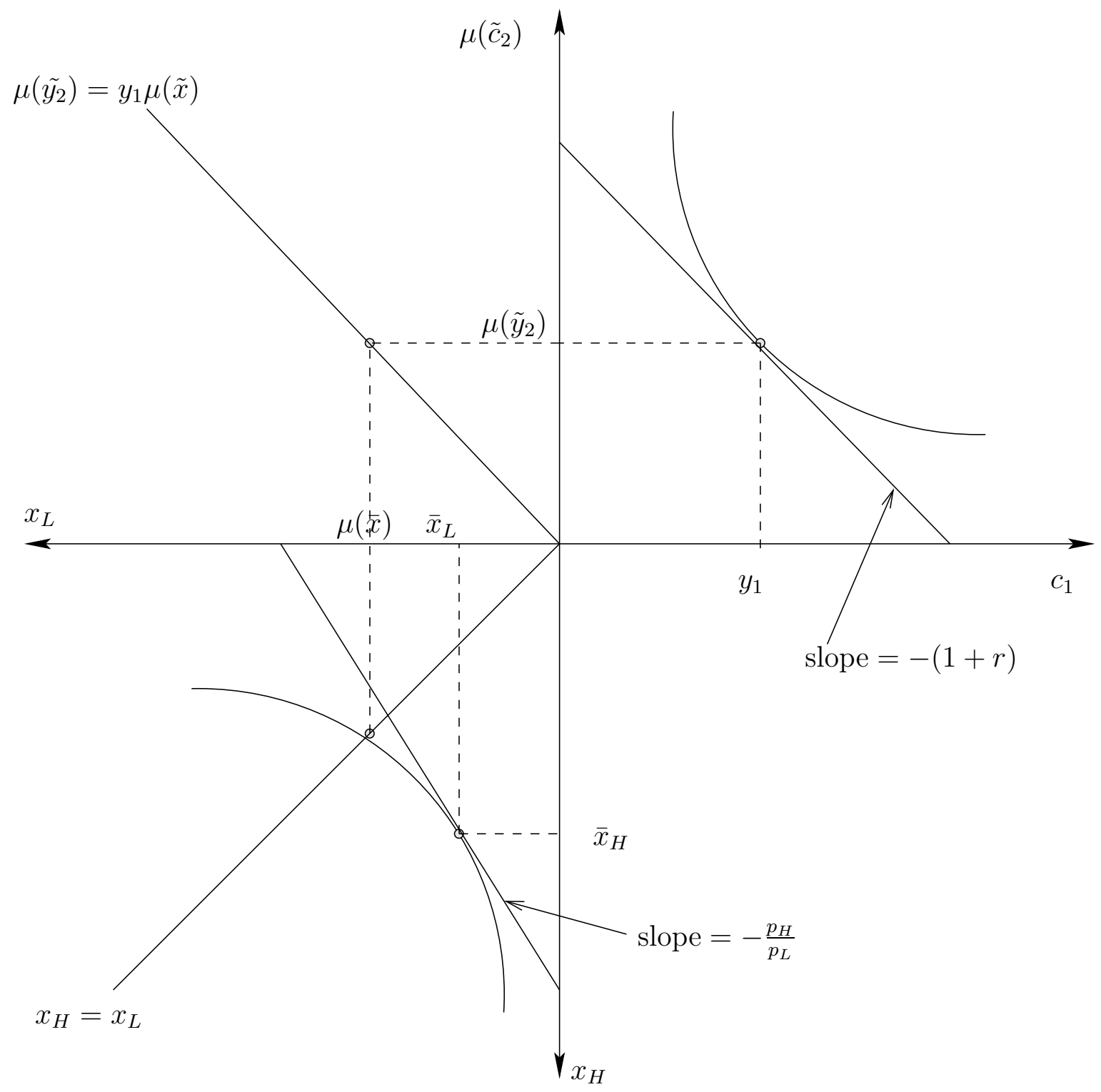

Two-period asset pricing economy. The lower left quadrant shows the trade-off over risky consumption growth rates. The upper right quadrant shows the intertemporal trade-off between certain consumption plans. The upper-left quadrant shows the connection between the certainty equivalence of the risky consumption growth to the intertemporal consumption. 
Figure 8: Two-Period Equilibrium - Disappointment Aversion

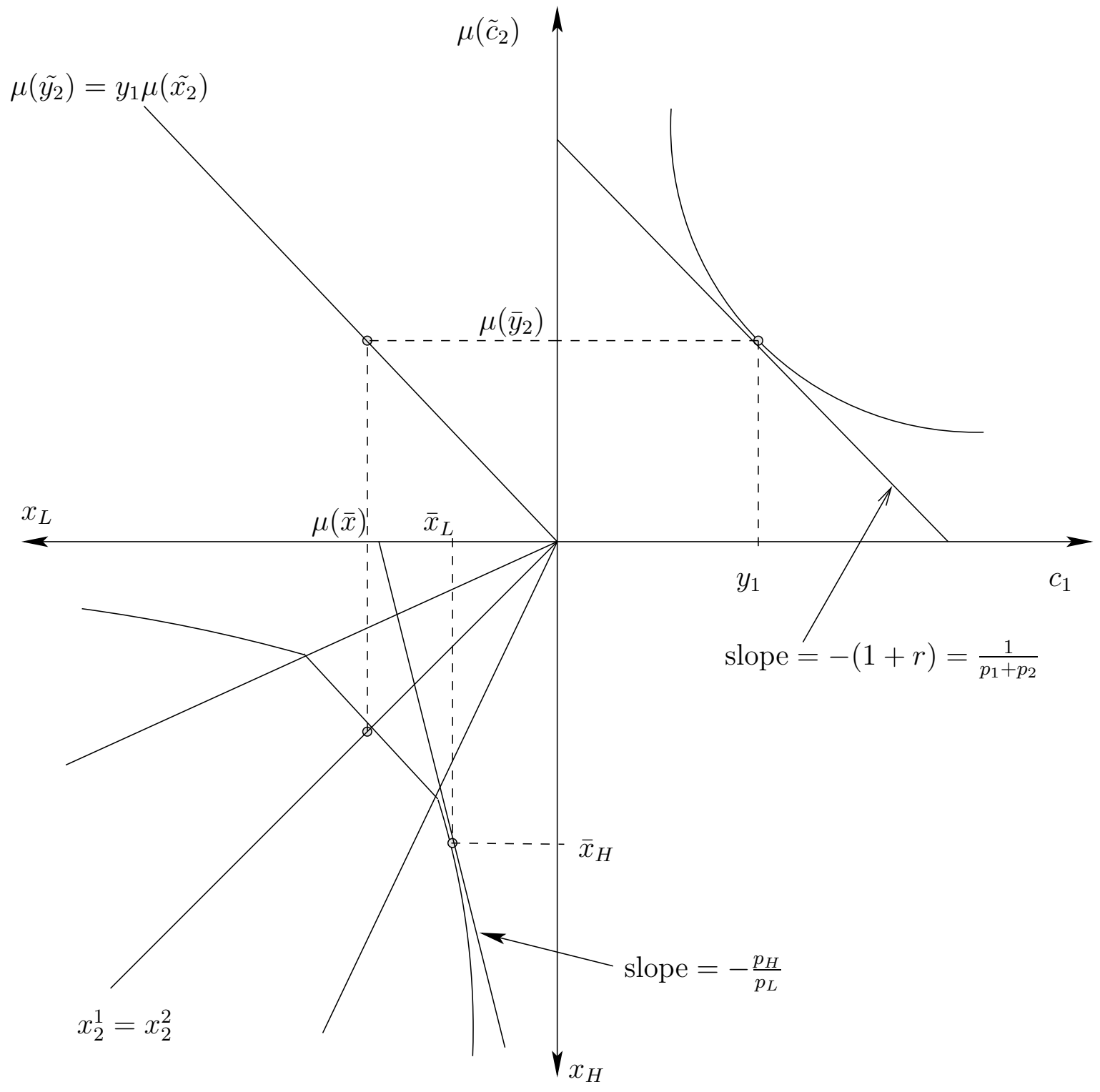

Two-period asset pricing economy. The lower left quadrant shows the trade-off over risky consumption growth rates. The upper right quadrant shows the intertemporal trade-off between certain consumption plans. The upper-left quadrant shows the connection between the certainty equivalence of the risky consumption growth to the intertemporal consumption. 